\title{
A Review of Risk Identification of Multinational Agricultural Products Supply Chain
}

\author{
Bo YANG ${ }^{a}$ and Han-Meng SU ${ }^{\text {b, }}$ \\ JiangXi University of Finance and Economics School of Information Management Nanchang, China \\ ayb_lh@163.com, bsuedyx@163.com \\ ${ }^{*}$ Corresponding author
}

Keywords: Transnational agricultural product supply chain, Supply chain risk management, Research review.

\begin{abstract}
This paper systematically combings and reviews the risk factors, risk identification of multinational agricultural products supply chain, and attempts to construct a theoretical framework for the research on risk management of multinational agricultural product supply chains. Finally, this paper proposes a research idea based on the theory of biological immunology to study risk identification models and supporting methods for multinational agricultural product supply chains.
\end{abstract}

\section{Introduction}

Supply chain risk refers to the fragility of the supply chain: due to the occurrence of risk factors, the supply chain's operating efficiency is reduced, the cost is increased, and even the property of the whole is broken. Da Qingli et al. [1] believe that any node company in the supply chain has problems and will affect the entire supply chain, while other node companies cannot prevent the risk of individual companies.

Multinational agricultural products supply chains refer to the combination of agricultural products supply chain worldwide. With a global perspective, the supply chain of agricultural products is extended to the scope of multinational cooperation. The cross-border agricultural supply chain faces an intricate network structure. The internal node companies have revenue distribution and risk sharing conflicts, which make them have the risk characteristics of the domestic agricultural product supply chain, and also have obvious complexity features and secondary derivative hazards. Therefore, it is necessary to sort out the research status of multinational agricultural product supply chain risk management. This article sorts out the risk factors and risk identification of multinational agricultural product supply chain, and draws on the principle of biological immunity, and proposes the research ideas on the research of multinational agricultural product supply chain risk identification models and supporting methods based on biological immunology theory.

\section{A Review of Risk Identification Research}

Risk identification refers to the sources of risk that people use in systematic and continuous understanding of various methods before the occurrence of risk accidents, and the potential causes of the analysis of risk accidents.

\section{Risk Identification Study}

In recent years, scholars at home and abroad have paid close attention to the qualitative analysis of supply chain risk sources and hazards, have classified the possible risk factors in the supply chain. Fan Xing [2] proposed that for the entire supply chain, in addition to the internal risk generated in the supply chain and the risks generated by the external environment, there should also be risk factors and convergence risks arising from intermediate links, such as logistics risk and exchange rate risk, which are all multinational supply chains. This paper takes the multinational agricultural 
product supply chain node enterprises as the research object, and divides the main risks into the following: internal risk, exogenous risk and intermediate link risk.

1. Enterprise endogenous risk

Enterprise endogenous risk refers to the risk factors generated by internal disharmony. It is mainly divided into three aspects: enterprise management risk, production risk and information risk.

(1)Enterprise management risk: Enterprise management risk mainly refers to the imbalance of the response mechanism. Zhang Yuchun believed that the main factors affecting the company's rapid response were the degree of process integration in the supply chain, integration of information and logistics, and customer demand orientation.

(2)Production risk: Due to the special nature of agricultural products, production risk is an important part of the risk factors of multinational agricultural product supply chains. It mainly refers to the impact of purchasing risk, inventory risk and the risk aversion of farmers in the uncertain environment on the whole supply chain.

(3)Information risk: Information risk has always been a hot issue studied by scholars at home and abroad. It refers to the process of distortion of effective information in the supply chain circulation. It can be divided into the risks brought about by the hidden information of the partners and the risks brought by the poor circulation of information within the company. Da Qingli [1] believes that the bullwhip effect caused by poor demand forecasting has caused enterprises to pay excess raw material and other costs, which will have a serious impact on the benefits of the company and even the economy of the entire country. From Figure 2, it can be seen that information risk exists in all aspects of the supply chain transmission, not just the enterprise endogenous risk.

2. Enterprise exogenous risk

Enterprise exogenous risk refers to the risk factors arising from the external environment of the supply chain node enterprise. It is mainly divided into four aspects: supply risk, environmental risk, demand risk and partner risk [2].

(1)Supply risk and environmental risk: Supply risks often coexist with environmental risks. The complex nature of supply risks in multinational agricultural product supply chains involves many subjects and many links, and the unique natural property of agricultural products that are susceptible to spoilage makes the risk control more difficult. Tassos Haniotis studied the coordination model of a two-stage agricultural supply chain system consisting of a risk-averse producer and a risk-neutral company under the circumstances that non-catastrophic weather affects agricultural output and transportation. The results indicate that when the degree of adverse environmental conditions exceeds a certain level, the user's purchasing enthusiasm will be affected, which in turn will cause demand risks.

(2)Demand risk: The demand risk mainly means that the demand of a few large customers is difficult to predict; the demand caused by promotions or unsalable sales fluctuates greatly; the improper delivery schedule causes the delivery of important customers to be affected; the shortage of important customer demand due to inventory shortage is low; the seasonal fluctuations in demand cannot be predicted; the customer financial conditions deteriorate; the new products are listed frequently and quickly. Uncertainty of demand is one of the important issues faced by inventory managers. Under a volatile market environment, the higher the uncertainty of demand, the greater the difficulty in balancing supply and demand.

(3)Partner risk: Partner risk refers to the risk of leakage of partner information due to the interdependence among partner companies. J. Hallikas et al. think that the risks between enterprises are caused by external and human risks that lead to the failure of organizational operation. They also pointed out that the more a node enterprise in a supply chain enterprise node is dependent on another node enterprise, the greater the risk to the supply chain as a whole.

3. Intermediate link risk

Intermediate risk refers to the risks arising from the transaction and cooperation between the nodes in the supply chain operation, which is mainly about the risk of convergence, exchange rate risk and logistics risk [2]. 
(1) Linking risk: The risk of convergence is mainly structural imbalance risk and national policy risk, and so on. Du Xiaojun believes that policy risk is the convergence risk that multinationals can only passively adapt and cannot actively use. Therefore, for multinational companies in China, joint ventures are the better choice, but considering the subjective initiative of multinational companies, when enterprises enter the host country as sole proprietorship, they have a greater advantage. Ding Xiaochao believes that the structural imbalance of the supply chain system is the result of the unbalanced response mechanism and the distrust of partners. The superposition of risk factors makes the entire supply chain more irreversible.

(2) Exchange rate risk and logistics risk: Different from the risk of domestic agricultural product supply chain, the risk factors in the supply chain of transnational agricultural products often need to consider the influence of exchange rate change and inflation factors [2]. In an international logistics outsourcing problem, enterprises generally consider macroeconomic factors as exchange rate, tariff and domestic tax rate. These factors can lead to changes of production plans. A. El Mokrini, E [3] believes that logistics outsourcing has become an important form of development in the new era, but risks and opportunities coexist, and companies need to take certain measures before they can maximize the benefits of the entire supply chain.

In summary, the author sorted out the overall framework diagram of the risk identification system for multinational agricultural product supply chains as shown in Figure 1. The figure shows the overall framework of a three-tier multinational agricultural product supply chain risk factor identification system consisting of domestic suppliers, foreign distributors and multinational companies.

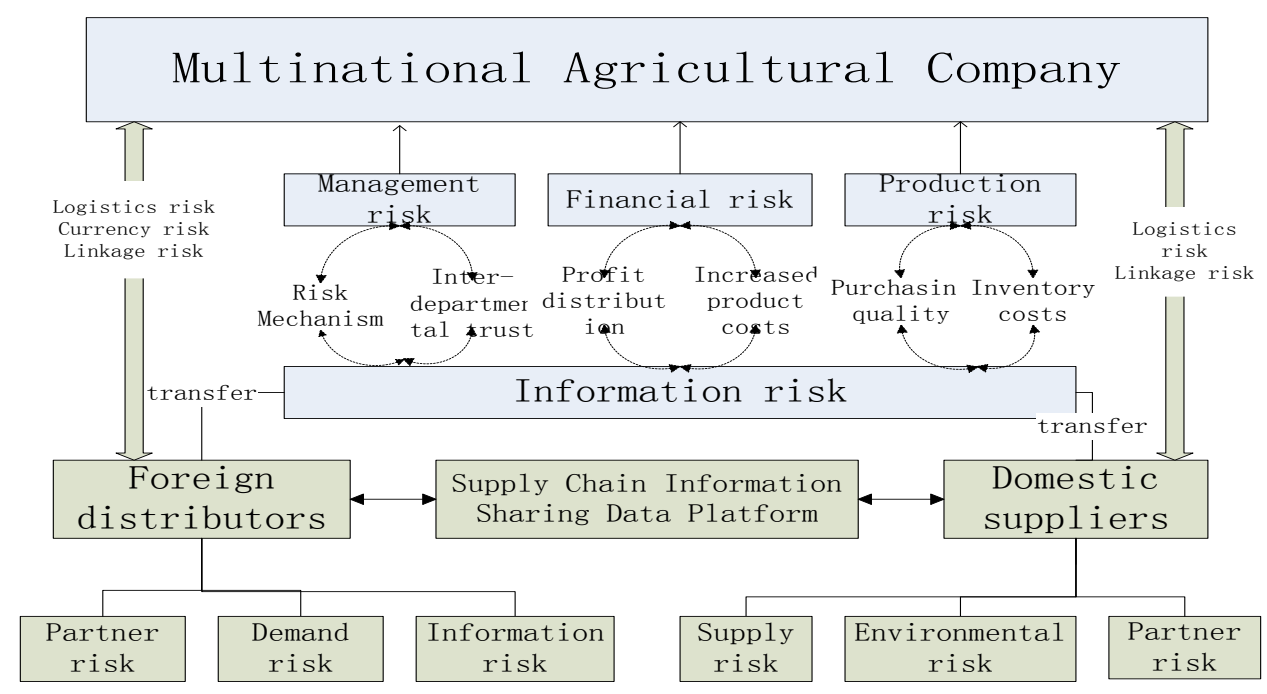

Fig. 1 The overall framework diagram of risk identification system for multinational agricultural product supply chain

\section{Research On Risk Identification Model}

When the historical data is sufficient, a specific risk factor probability distribution map can generally be established based on the risk identification model. For example, Analytic Hierarchy Process (AHP): It is necessary to describe risks hierarchically, build an evaluation system, and determine the weight of indicators by experts. Its advantages are simple and convenient and the disadvantage is that it cannot adapt to the dynamic and variability of risk factors due to environmental changes and insufficient historical data. Quantitative: Ding Weidong uses fuzzy risk factor analysis to identify risks, and evaluates risk factors in a fuzzy evaluation, which can clearly reflect the reliability of the supply chain system. On this basis, Xiao Wenzhen [4] used a multi-level fuzzy comprehensive evaluation method to deal with difficult-to-quantify risk factors systematically and clearly, making it intuitive to see the urgency of risk factors.

To sum up, first of all, the research on the risk identification of agricultural product supply chain has been relatively mature, but the research on the conduction and dynamic nature of risk factors is relatively weak. Secondly, past studies have mostly focused on the analysis of the operational status 
of a single or several links in the supply chain, and there is a lack of research on the risk of convergence in the supply chain. However, the risk of cohesive links not only exists, but also is an inevitable risk factor. Thirdly, there is no memory mechanism in the risk identification system. Therefore, when the same risk factor occurs, it needs to identify the risk again, resulting in waste of resources.

\section{Future Research Prospects}

In general, faced with the dynamic risk factors existing in the external environment, supply chain node enterprises generally adopt the strategy of "taking change with change". But the fact is that the structural changes in the supply chain are difficult to get ahead of the environment and it is even difficult to catch up with changes in the environment. So how to improve the anti-risk ability of multinational agricultural product supply chain? How to achieve "static strain" can flexibly respond to environmental changes? The existing research on the supply chain dynamic risk identification model is obviously insufficient.

Regarding future research directions, combined with the principle of biological immunity, the author believes that the following issues are worth studying:

1. Dynamic risk identification

The external environment faced by the multinational agricultural product supply chain is dynamic and continuous, but the change of the supply chain with the environment is a process of accumulation from qualitative change to quantitative change. It is a leap-forward development adaptation. Figure 3 is a sketch map of the supply chain adaptation to the environment. Therefore, the study of the dynamic and superposition of risk issues in multinational agricultural product supply chains will be a new perspective for the study of such issues.

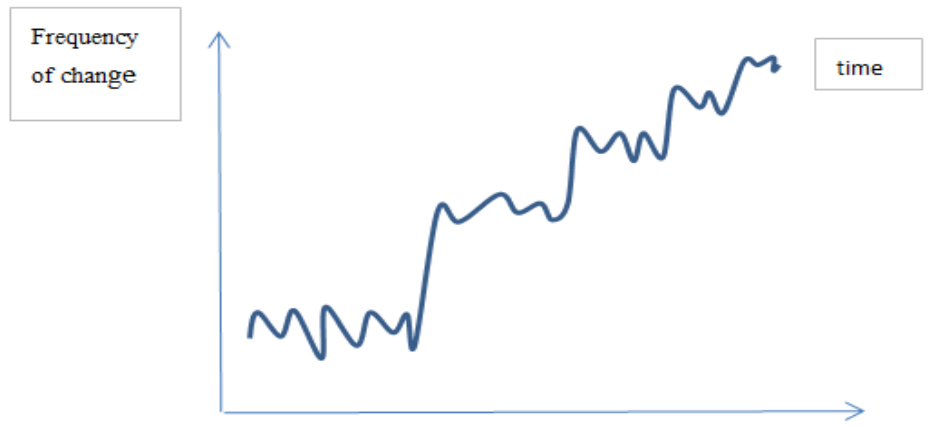

Fig. 2 The adaptive response of the supply chain

2. The memory and feedback mechanism of risk identification model

At present, almost all risk identification models are only applicable when risk factors occur, and they are screened as the first object of study. However, risk factors of the same mechanism of action often occur in all aspects of the supply chain. From Figure 2, it can be seen that the time required for the jump response of the supply chain to the external environment over time is getting shorter and shorter. This indicates that when the supply chain operates, there are internal memory functions for the occurrence of external risk factors. When the risk factors of the same result happen again, the supply chain companies' response time is shrinking. Therefore, the multinational agricultural product supply chain risk identification system requires a memory and feedback mechanism. It combines the individual risk identification activities once and again into a risk identification system.

3 . The combination of biological immune principle and risk identification

To make the supply chain risk management system effectively prevent unknown potential risk factors, Yang Qing [5] believes that it can learn from the research results of biological immunity theory and study unconventional emergencies from the perspective of multidisciplinary comprehensive integration. The principle of biological immunity refers to the sum of the biological effects produced by the collective in response to self and alien identification. In the future, the author believes that a multinational agricultural product supply chain risk management system can 
be studied through the understanding of the mechanisms and characteristics of biological immune responses. Because the principle of biological immunity is defensive, self-stabilizing, monitoring and learning, the combination of biological immune principles and risk identification has the following advantages:

(1) Memory and feedback will be greatly improved. The recognition mechanism of the risk identification system in the existing research results is mostly reflected in the environment. However, due to the timeliness of agricultural products, seasonality, and the bullwhip effect of information transmission, the occurrence of risk identification behavior should precede environmental changes. Because the immune system can deal with the source of risk, it can produce a memory and feedback system. This new system can suppress the recurrence of the crisis [6]. Because of the defensive experience, the immune behavior of the supply chain can be advanced.

(2) The risk factors can be monitored in real time. The immune system has self-learning, evolution, and monitoring capabilities, which can help the supply chain risk management system to eliminate "dissident" factors at the first time.

Therefore, the comprehensive use of immunological theory to study the risk identification of multinational agricultural product supply chain has a very broad and in-depth research space.

\section{Acknowledgements}

This work was financially supported by the National Natural Science Foundation project (71640022, 71561010), the Jiangxi Province Social Science "Twelfth Five Year Plan" project(15TQ04).

\section{References}

[1] Da, Zhang Qin, Shen Houcai. Study on the problem of bullwhip effect in supply chain [J].Journal of management science, 2003, (03): 86-93

[2] Fan Xing, Shao Juping, sun Yanan. Risk identification and evaluation of transnational agricultural products supply chain based on fuzzy theory [J].Research on science and technology management, 2016, 36 (06): 210-215.

[3] A. El Mokrini,E. Dafaoui,A. Berrado,A. El Mhamedi. An approach to risk Assessment for Outsourcing Logistics: Case of Pharmaceutical Industry[J]. IFAC PapersOnLine,2016,49(12):.

[4] Xiao Wenzhen, Liu Zhenyuan, Zhong Weihua. Multilevel fuzzy comprehensive evaluation of the risk of engineering supply chain [J]. technology progress and countermeasures, 2010,27 (19): 158-160.

[5] Yang Qing, Liu Xingxing, Chen Ruiqing, Cai Wentao.An immune system based risk identification model for unconventional emergencies [J].Journal of management science, 2015,18 (04): 49-61.

[6] Wang Yihua, Lv Ping, Xu Bo, Yang Zhenning, Su Xiaoyang, Du Debin, Song Zhicheng, duer Xingguo. Preliminary research on tissue immunity [J]. science and technology management, 2006 (06): 133-139. 